Research report

\title{
The influence of different Stop-signal response time estimation procedures on behavior-behavior and brain-behavior correlations
}

\author{
C. Nicolas Boehler ${ }^{\mathrm{a}, \mathrm{b}, *}$, L. Gregory Appelbaum ${ }^{\mathrm{a}, \mathrm{c}}$, Ruth M. Krebs ${ }^{\mathrm{a}, \mathrm{b}}$, \\ Jens-Max Hopf ${ }^{\mathrm{d}}$, Marty G. Woldorff ${ }^{\mathrm{a}, \mathrm{c}}$ \\ a Center for Cognitive Neuroscience, Duke University, Durham, NC, USA \\ ${ }^{\mathrm{b}}$ Department of Experimental Psychology, Ghent University, Belgium \\ ${ }^{c}$ Department of Psychiatry, Duke University, Durham, NC, USA \\ d Department of Neurology, Otto-von-Guericke-University, Magdeburg, Germany
}

\section{A R T I C L E I N F O}

\section{Article history:}

Received 4 September 2011

Received in revised form 5 December 2011

Accepted 2 January 2012

Available online 8 January 2012

\section{Keywords:}

Inhibitory motor control

Stop-signal task

Stop-signal response time

Brain-behavior correlations

Human

\begin{abstract}
A B S T R A C T
The fundamental cognitive-control function of inhibitory control over motor behavior has been extensively investigated using the Stop-signal task. The critical behavioral parameter describing stopping efficacy is the Stop-signal response time (SSRT), and correlations with estimates of this parameter are commonly used to establish that other variables (e.g., other behavioral measures or brain activity measures) are closely related to inhibitory motor control. Recently, however, it has been argued that SSRT estimates can be strongly distorted if participants strategically slow down their responses over the course of the experiment, resulting in the SSRT no longer reliably representing response-inhibition efficacy. Here, we performed new analyses on behavioral and functional data from an fMRI version of the Stop-signal task to gauge the consequences of using different SSRT estimation approaches that are differentially prone to the influence of strategic response slowing. The results indicate that the SSRT estimation approach can dramatically change behavior-behavior correlations. Specifically, a correlation between the SSRT and Go-trial accuracy that was highly significant with one estimation approach, virtually disappeared for the other. Additional analyses indeed supported that this effect was related to strategic response slowing. Concerning brain-behavior correlations, only the left anterior insula was found to be significantly correlated with the SSRT within the set of areas tested here. Interestingly, this brain-behavior correlation differed little for the different SSRT-estimation procedures. In sum, the current results highlight that different SSRT-estimation procedures can strongly influence the distribution of SSRT values across subjects, which in turn can ramify into correlational analyses with other parameters.
\end{abstract}

(C) 2012 Elsevier B.V. All rights reserved.

\section{Introduction}

The ability to suppress unwanted behavioral responses is critical for successful goal-directed behavior, as it allows individuals to quickly adjust to a changing environment by overcoming pre-potent responses when they are inadequate or inappropriate (see e.g., [1] for a review). Interest in this topic has dramatically increased over the past several years in accord with the

Abbreviations: IFG, inferior frontal gyrus; pre-SMA, pre-supplementary motor area; SSRT, Stop-signal response time; SSRTi, SSRT estimated by the integration approach; SSRTm, SSRT estimated by the mean approach; SST, successful Stop-trial; UST, unsuccessful Stop-trial.

* Corresponding author at: Department of Experimental Psychology, Ghent University, Henri Dunantlaan 2, 9000 Ghent, Belgium. Tel.: +32 926464 64; fax: +32 92646496

E-mail address: c.n.boehler@gmail.com (C.N. Boehler). fundamental role of inhibitory functions in normal human behavior and development, as well as in a variety of neurological and psychiatric conditions, including attention-deficit hyperactivity disorder (ADHD) and substance abuse [2-6].

Typically, these inhibitory functions are assessed by means of versions of the Go-NoGo or Stop-signal tasks. The Stop-signal task in particular has received a lot of attention recently, partly because it is thought to be relatively robust against strategic adjustments by the participants. In this task a choice-reaction Go-stimulus is, on a minority of trials, rapidly followed by a Stop-stimulus that requires the participant to cancel the response to the Go-stimulus [7,8]. A wide range of neuroscientific approaches have yielded converging evidence that a mostly right-hemisphere network of brain areas is critical for response inhibition (but see [9]). This network includes the inferior frontal gyrus (IFG; particularly the frontal operculum extending into the insula) and the pre-supplementary motor area (pre-SMA) which are thought to interact with the basal ganglia, 
and the thalamus to inhibit a motor response (for reviews see $[4,5,10,11])$.

One of the key advantages of the Stop-signal task is its strong foundation in formal computational modeling $[5,7,12,13]$. Such explicit modeling permits the estimation of parameters that cannot be observed directly, such as the Stop-signal response time (SSRT), which estimates how quickly participants can cancel an already-initiated response (usually around $200 \mathrm{~ms}[7,14]$ ). Because this parameter has to be estimated from the distributions of Gotrial response times and the Go-stimulus-Stop-stimulus stimulus onset asynchronies (Go-Stop SOAs) on Stop-trials, a variety of estimation procedures have been developed (for reviews see $[15,16]$ ). In one commonly used approach, a tracking procedure is used during the experiment to dynamically adjust the Go-Stop SOA to yield $50 \%$ successful Stop-trials. Under such conditions the 'mean approach' can be used, which derives the SSRT by subtracting the mean Go-Stop SOA from the mean Go-trial RT (henceforth termed SSRTm here). An alternative approach is the so-called 'integration approach'. In contrast to the mean approach, the integration approach can be used, not only in the presence of a successful tracking procedure, but also if the stopping-success rate is not $50 \%$. To achieve this flexibility, the Go-trial RTs are rank-ordered, and the RT value at the percentile that corresponds to the percentage of failed inhibitions is determined on a per-subject basis (e.g., the RT at the 61 st percentile of the Go-RT distribution for a participant with $61 \%$ unsuccessful Stop-trials). The SSRT is then estimated by subtracting the average Go-Stop SOA from this RT value (henceforth termed SSRTi). Alternatively, the corresponding RT percentile can be determined for all (or for the central) Go-Stop SOAs and the respective results are averaged. Nonetheless, as the real SSRT is unknown, and both of the above approaches (and others) are only estimating procedures, it remains an open question as to which approach should be used. ${ }^{1}$

Notably, it has recently been argued that strategic/motivational aspects of performing the Stop-signal task can systematically distort the estimate of the SSRT [17], in particular if they result in more than $50 \%$ successful Stop-trials. Such a deviation from an even ratio can result from strategic response slowing throughout the experiment (i.e., when participants try to maximize their number of successful Stop-trials by ignoring the instruction to always respond quickly to the Go-stimuli), even in the presence of an adaptive procedure that adjusts the Go-Stop SOA based on the participants' performance. Considering that such slowing can easily occur in these sorts of studies, this potential SSRT-estimation inaccuracy is important to take into account. From a theoretical perspective, the SSRTi estimate should be more robust against such influences than simple versions of the SSRTm because it takes into account variations in the ratio of successful Stop-trials. Here we perform new analyses on data from a recent study in which we used an adapted version of the Stop-signal task that, as a sensory control condition, included task-blocks where the Stop-stimuli were irrelevant to the task (Fig. 1; [18]). In our earlier report, we used the mean approach to estimate the SSRT (SSRTm) and found

\footnotetext{
${ }^{1}$ In order to investigate this heterogeneity, we have looked at the sample of Stopsignal tasks in a recent meta analysis that reviewed functional-imaging studies on that topic [11]. The meta analysis contained 21 studies that employed the Stopsignal task. Of those, three did not report an SSRT estimate. Further idiosyncrasies aside, eleven of the remaining studies used the average of the Go-Stop SOA values for the calculation, of which four used the mean approach, four the integration approach (note that three of those studies did not use an adaptive staircase procedure), and three more the median approach (the same as the mean approach but using the median of the Go-RT distribution instead). The remaining seven studies used variants of the mean or median approach but did not use the full range of Go-Stop SOA values for the estimation (i.e., these studies either waited for the Go-Stop delay staircase to stabilize or tried to estimate the specific Go-Stop SOA at which the probability of a successful inhibition was exactly $50 \%$ ).
}

\section{A Stop-relevant blocks}
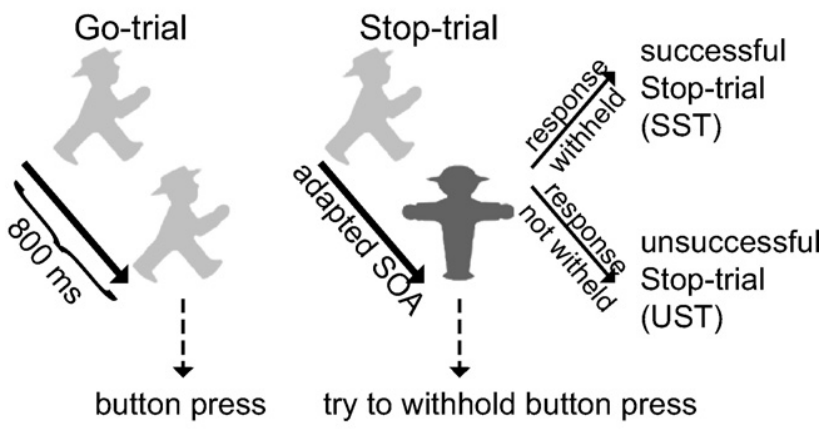

B

\section{Stop-irrelevant blocks}
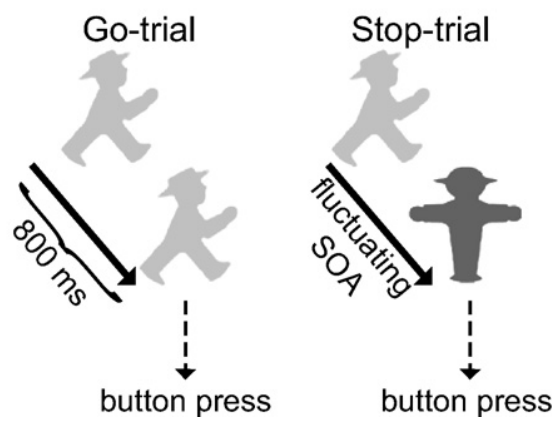

Fig. 1. Paradigm. (A) In Stop-relevant blocks, a choice-reaction stimulus (a green German traffic-light symbol oriented to the left or right-here represented in light grey-requiring an index/ring-finger response respectively) was either presented for $800 \mathrm{~ms}$ (Go-trial) or rapidly replaced by a red Stop-stimulus (here represented in dark grey; Stop-trial). This Stop-stimulus was presented after a variable SOA set by a tracking algorithm and indicated that the response to the Go-stimulus on that trial was to be inhibited, thereby yielding successful (SST) and unsuccessful Stoptrials (UST). (B) In Stop-irrelevant blocks the visual stimulation was identical, but the Stop-stimuli were irrelevant and behavioral responses were required on all trials, thus providing a sensory baseline condition and a reference for response speed in the absence of task-relevant Stop-stimuli.

a strong negative correlation with general task accuracy (most importantly with Go-trial accuracy), indicating that participants with high Go-trial accuracy were also better inhibitors (i.e., had shorter SSRTs). Interestingly, SSRTm and task accuracy both correlated with activity estimates in the left anterior insula.

Based on the concern that strategic slowing might have affected our results, we wanted to directly assess the influence of SSRTestimation procedures on across-subject correlational analyses between this parameter and others, including behavioral parameters that estimate the tendency of participants to strategically slow their Go-trial responses in a Stop-signal task. Beyond general differences in behavior-behavior and brain-behavior correlations between the SSRTm and the SSRTi, we were particularly interested in whether the aforementioned correlation between SSRTm and task accuracy in this dataset [18] might have been related to the influence of strategic response slowing on the SSRTm estimate. Specifically, if different degrees of response slowing biased our SSRTm estimates, it would be conceivable that this same response slowing could have also ramified into higher Go-trial accuracy. Such an influence on both variables might thus explain the strong correlation between the two, in that they would both be attributable to strategic slowing. If that were the case, the additional question would arise whether the brain-behavior correlation between insula activity and SSRTm that we also reported in this earlier publication would be affected in a similar fashion. 


\section{Experimental procedure}

\subsection{Participants}

Eighteen participants took part in this study, of whom two had to be excluded due to technical problems, and another one due to poor behavioral performance, thereby resulting in fifteen participants that were included in the analyses ( 9 female; mean age 22.9 years). All participants had normal or corrected-to-normal visual acuity, and did not report a history of psychiatric or neurological disorders. Written informed consent was obtained before the experiment in accordance with the Duke Institutional Review Board and participants were compensated for their participation at the rate of $\$ 20$ per hour.

\subsection{Task}

The present experiment entailed two variants of the typical Stop-signal task [14]. Stop-relevant blocks represented the typical Stop-signal task in that participants were instructed to try to withhold their response to the Go-stimulus when a Stopstimulus occurred, whereas in Stop-irrelevant blocks the visual stimulation was identical, but participants were instructed to ignore the Stop-stimuli (i.e., to always respond $[18,19])$. Each task was performed once per experimental run (approximately 2.5 min each per run), separated by a 16 -s break. Odd runs began with the Stop-relevant task, even runs with the Stop-irrelevant task. Ten runs were collected for each participant, yielding a total of 943 trials per participant. The trial onset asynchrony was varied pseudo-randomly between 2 and $8 \mathrm{~s}$ (gamma distribution; average $3.2 \mathrm{~s}$ ) to allow for the separation of different conditions in an event-related fMRI analysis [20].

Stop-relevant blocks used a standard Stop-signal task (Fig. 1A) with a random sequence of Go-trials ( $80 \%$ of trials) and Stop-trials ( $20 \%$ of trials). On Go-trials, the orientation of a Go-stimulus had to be discriminated (oriented to the left: right index finger; oriented to the right: right middle finger). On Stop-trials, the Go-stimulus was quickly followed by a Stop-stimulus, indicating that the response to the Gostimulus was to be canceled, yielding successful (SST) and unsuccessful Stop-trials (UST). Total stimulus duration was always $800 \mathrm{~ms}$. During Stop-trials, the Go-Stop SOA was titrated using an adaptive staircase procedure to yield similar numbers of SST and UST for each participant. The staircase procedure increased the SOA by $17 \mathrm{~ms}$ after SST and decreased it by the same amount after UST (initial SOA: $200 \mathrm{~ms}$ ).

Visual stimulation during Stop-irrelevant blocks was identical to that of Stoprelevant ones (Fig. 1B), but participants were instructed to respond to all Go-stimuli irrespective of the occurrence of Stop-stimuli. To maximally equate the sensory stimulation between the two block types, we also varied the Go-Stop SOA in Stopirrelevant blocks. Specifically, SOA values were varied in a random one-up/onedown fashion after each Stop-irrelevant Stop-trial within \pm three 17-ms steps of the initial value. All staircases used the end value of the preceding Stop-relevant block as the start value.

\subsection{Behavioral analysis}

The key parameter describing stopping efficacy that is typically derived in a Stop-signal task is the SSRT. It describes by how much the presentation of the Stopstimulus needs to precede the moment when a response would be executed so that this response can still be canceled, i.e. how much time the brain needs to implement response cancelation. Here, we used two different procedures to estimate this parameter: the mean approach (yielding what we term the SSRTm) derives the SSRT by subtracting the mean Go-Stop SOA from the average Go-trial response time [8]. This procedure has been argued to be the most reliable approach for estimating the SSRT, if (and only if) the ratio of successful and unsuccessful Stop-trials is $50 \%$ [16]. However, small deviations from this even ratio can occur even in the presence of a tracking procedure that adjusts the Go-Stop SOA to different Go-trial response speeds. To directly address the implication of this potential issue here, we additionally estimated the SSRT using a version of another common approach, the integration approach [14], yielding an estimate we label SSRTi. Here, Go-trial RTs are rank-ordered, and the RT value at the percentile that corresponds to the percentage of unsuccessful Stop-trials is determined on a per-subject basis (e.g., 61 st percentile of the Go-RT distribution for a participant with $61 \%$ unsuccessful Stop-trials). The average Go-Stop delay is then subtracted from this Go-RT value. By taking deviations from an even ratio between successful and unsuccessful Stop-trials into account, this approach is more robust against such variations. Note, however, that other methods for estimating the SSRT exist, including some approaches that might be less prone to strategic response slowing as well. Such approaches include versions of the mean approach that aim to limit the Go-Stop SOAs to a subset of values that lead to a $~ 50 \%$ stopping success, rather than simply averaging the full range of Go-Stop SOAs [16].

Since strategic response slowing was of prime interest in this study, we endeavored to quantitatively characterize this parameter for each participant. Similarly to the study by Leotti and Wager [17], we derived two different parameters to describe this aspect: (1) as a measure of average response slowing due to task-context, we subtracted the average Stop-irrelevant Go-trial RT from their Stop-relevant-block counterpart. This parameter is closely related to pro-active response slowing (e.g., [21]; a term we will use here, which has been used to describe the tendency of subjects to generally slow down their Go-trial responses in the context of Stop-trials).
(2) We computed how much participants slowed down their Stop-relevant-block Go-trial responses over the course of the experiment. Such ongoing slowing is in fact necessary to achieve a high proportion of successful Stop-trials in that it is the only way to avoid the staircase algorithm "catching up" and increasing the Go-Stop SOA sufficiently to counteract slow Go-responses. This parameter was estimated by computing the difference in Stop-relevant-block Go-trial RT from the second minus the first half of the experiment (here termed progressive response slowing).

Across-subject, behavior-behavior correlations were calculated between the following parameters: SSRTi, SSRTm, proportion of successful Stop-trials, Stoprelevant-block Go-trial accuracy, pro-active response slowing, and progressive response slowing. Statistical analyses of the behavioral data were performed using Pearson's correlation. One-tailed $p$-values are reported because clear predictions could be made for the direction of the key correlations (e.g., that response slowing leads to shorter SSRTm estimates and to more accurate Go-trial responding, etc.). In order to statistically compare them, we transformed correlation coefficients into $z$ scores and estimated the difference between coefficients in relation to the standard error of this difference.

\subsection{FMRI data acquisition and analysis}

MR data was acquired on a 3-Tesla GE Signa MRI scanner. Functional images were acquired with a reverse-spiral sequence $(T R=2000 \mathrm{~ms}, T E=25 \mathrm{~ms}$; flip angle $=75^{\circ}$; 32 slices with $3 \mathrm{~mm} \times 3 \mathrm{~mm} \times 3 \mathrm{~mm}$ resolution; AC-PC orientation covering the brain from the top approximately down to the Pons). To reach steady-state magnetization, the first five functional images of each run were discarded. Additionally, a high-resolution structural T1 (3D Fast Spoiled Gradient Recalled (FSPGR); $1 \mathrm{~mm} \times 1 \mathrm{~mm} \times 1 \mathrm{~mm}$ resolution) was obtained. Data were analyzed using SPM5 (http://www.fil.ion.ucl.ac.uk/spm/) including the following steps: correction for acquisition time delay; spatial realignment; spatial normalization (applying the normalization matrix used to warp the individual anatomical images onto the SPM template); reslicing to a voxel size of $2 \mathrm{~mm} \times 2 \mathrm{~mm} \times 2 \mathrm{~mm}$; and spatial smoothing (8-mm full-width half-maximum Gaussian kernel). First-level statistical modeling was based on canonical hemodynamic response functions (HRF) combined with time and dispersion derivatives, while additionally applying a 128-s high-pass filter [22]. All major conditions were modeled separately and restricted on trials with correct responses (or no response for successful Stop-trials). Additional regressors modeled trials with incorrect responses, misses, the onsets of breaks, as well as the six spatial realignment parameters.

The parameter estimates resulting from each condition/contrast and participant (first-level analysis) were entered into a second-level, random-effects group analysis using one-sample $t$-tests. For visualization purposes, activation maps were rendered on the SPM single-subject template. Since this report is a follow-up on our earlier work and is based on portions of the same dataset [18], the only brain-activity contrast used here for correlations with behavioral parameters is the betweenblock conjunction that was used for brain-behavior correlations in that earlier report. Specifically, a conjunction of successful and unsuccessful Stop-trials respectively compared against Stop-irrelevant Stop-trials (i.e., a sensory control without response inhibition) was performed and thresholded at $p<0.002$ with an extent threshold $k=10$ contiguous voxels ( $p<0.005$ and $k=5$ for subcortical activations; see Table 5 in [18]). The resulting local maxima were used for a region-of-interest (ROI) analysis, in which Marsbar (http://marsbar.sourceforge.net/) was used to extract beta weights from spherical 4-mm radius ROIs centered on those local maxima (see Table 5 in [18] for the full list of ROIs). As in our previous report, we extracted the differences of the beta values corresponding to the contrasts "SST vs. Stop-irrelevant Stop-trials" and "UST vs. Stop-irrelevant Stop-trials", and these two values were then averaged for each subject to capture the degree to which activity in these ROIs was more pronounced during Stop-relevant vs. Stop-irrelevant Stop-trials.

In order to probe brain-behavior relationships between activity levels in the different ROIs and the SSRT measures, we correlated the activity estimates extracted from the ROIs with the SSRTm, Go-trial accuracy (already reported in [18]), and SSRTi values across participants. One-tailed $p$-values are reported for these correlational analyses, in that we expected negative correlations with the SSRT estimates in areas related to stopping efficiency. Additional brain-behavior correlations based on the same data can be found in our earlier report [18].

\section{Results}

\subsection{Behavioral data}

Table 1 provides an overview of the response-time and accuracy parameters. During Stop-relevant Stop-trials, participants managed to withhold their behavioral response on approximately half of the trials, indicating that our staircase SOA-adjustment procedure had been relatively successful in this regard. Importantly, these values varied quite closely around 50\% ranging between $46 \%$ and $59 \%$ successful Stop-trials. The SSRT estimated by the two procedures yielded very similar average values. The mean approach 

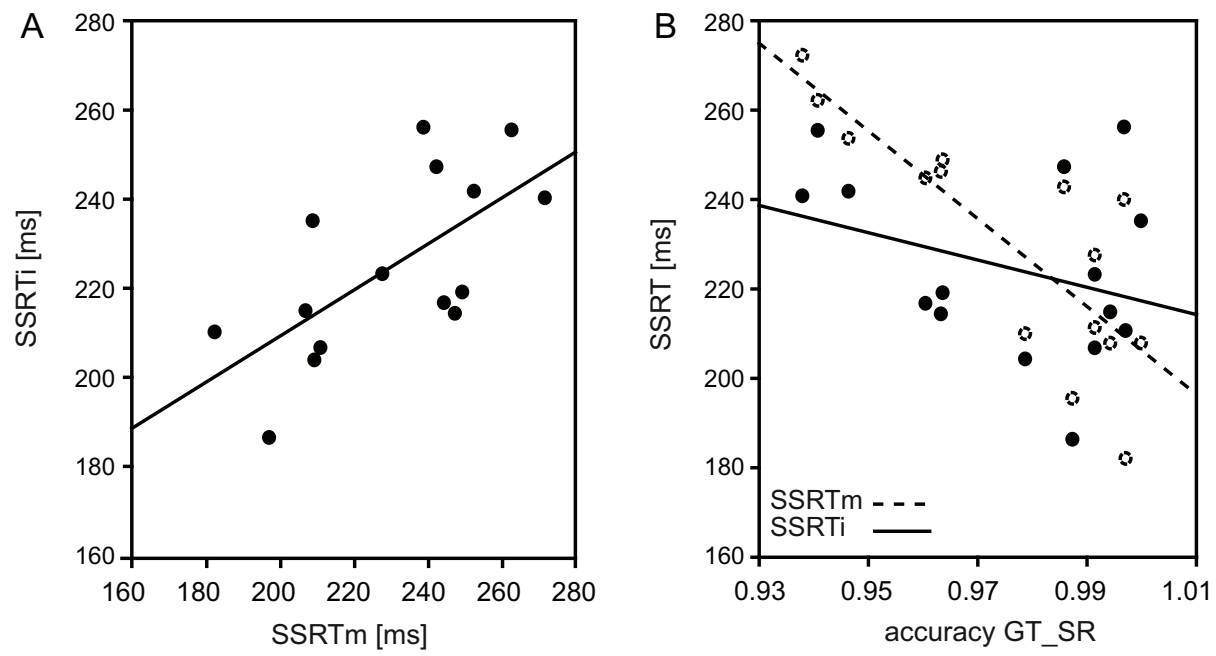

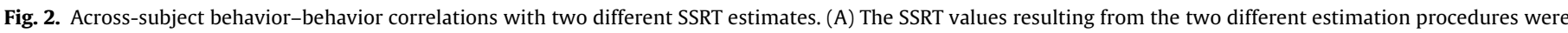

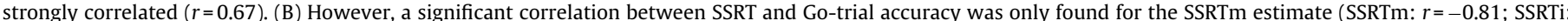
$r=-0.33$ ).

(SSRTm) yielded $230 \mathrm{~ms}$, whereas the integration approach (SSRTi) resulted in $225 \mathrm{~ms}$, which did not differ significantly (two-tailed paired $t$-test: $p>0.3$ ). Nevertheless, the two estimates appeared to differ somewhat within participants, as evidenced by a correlation coefficient of only 0.67 of the two estimates across participants (Fig. 2A). While highly significant $(p=0.003)$, this correlation is somewhat modest given that both variables estimate the same parameter and result in highly similar grand-average values.

As reported in our previous article using the mean SSRTestimation approach only [18], the SSRTm values correlated inversely with task accuracy in Stop-relevant-block Go-trials to a very high degree $(r=-0.81, p<0.001)$. In contrast, the SSRTi values calculated here produced a considerably different result (Fig. 2B), in that it did not yield a significant correlation with task accuracy $(r=-0.33 ; p>0.1$; see Table 2 for an overview of behavior-behavior correlations). Importantly, the two correlation coefficients differed significantly from one another (one-tailed $p=0.001$ ). One possible explanation for why the SSRTm correlates with Go-trial accuracy (and the SSRTi does not) could be related to the influence of strategic slowing on the SSRTm. Leotti and Wager [17] report that SSRT estimates are shorter for subjects that slow down their Goresponses over the course of the experiment in order to achieve more than 50\% successful Stop-trials. Responding more slowly, in turn, is likely to also lead to higher Go-trial accuracy. Since the SSRTi

Table 1

Behavioral data (average \pm SD).

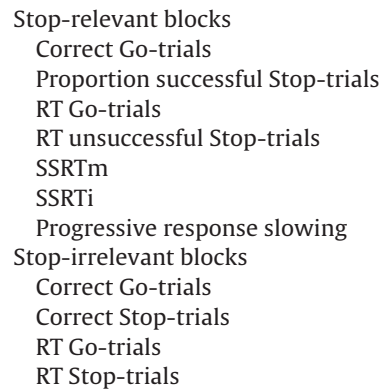

$97.6 \%( \pm 2.1)$ $52.7 \%( \pm 3.8)$ $520 \mathrm{~ms}( \pm 84)$ $446 \mathrm{~ms}( \pm 64)$ $230 \mathrm{~ms}( \pm 26)$ $225 \mathrm{~ms}( \pm 20)$ $4 \mathrm{~ms}( \pm 37)$

$96.6 \%( \pm 2.5)$ $97.1 \%( \pm 3)$ $436 \mathrm{~ms}( \pm 48)$ $439 \mathrm{~ms}( \pm 44)$

SSRTm = SSRT estimate using the mean approach; SSRTi = SSRT estimate using the integration method; progressive response slowing = Stop-relevant Go-trial RT from the 2 nd half of the experiment minus that of the first. estimate is less susceptible to such influences, the observation that it is not correlated with Go-trial accuracy is consistent with this notion.

To further investigate whether strategic slowing contributed to the correlation pattern described above, we performed additional analyses based on the two parameters we derived to describe strategic slowing (see Section 2.3). On average, participants slowed down their response by $84 \mathrm{~ms}$ during Stop-relevant blocks as compared to Stop-irrelevant blocks (pro-active response slowing). Additionally, we calculated whether subjects slowed down their Go-trial responses in Stop-relevant blocks over the course of the experiment by comparing Stop-relevant-block Go-trial RTs from the second minus the first half of the experiment (progressive response slowing). Although this value was not significantly different from zero across subjects (two-tailed one-sample $t$-test: $p>0.4$ ), there was a large degree of variation such that some subjects sped up while others slowed down their responses over the duration of the experiment.

Performing additional correlational analyses with these two indices of strategic slowing yielded some evidence in favor of the notion that strategic slowing (and the resulting higher proportion of successful Stop-trials) had some influence on the SSRTm, but not on the SSRTi. More specifically, the SSRTm was correlated with the proportion of successful Stop-trials $(r=-0.49 ; p=0.03)$, and there was a trend for a correlation with progressive response slowing $(r=-0.38 ; p=0.08)$. Neither correlation was present for the SSRTi (both $p>0.25$ ). In particular, the correlations with the proportion of successful Stop-trials yielded significantly different correlation coefficients for SSRTm and SSRTi (one-tailed $p=0.001$ ). Moreover, the correlation coefficients for progressive response slowing also differed significantly for the correlations with SSRTm and SSRTi (one-tailed $p=0.02$ ). Importantly, Stop-relevant Go-trial task accuracy was correlated with progressive response slowing $(r=0.62 ; p=0.007)$ and with the proportion of successful Stop-trials $(r=0.64 ; p=0.005)$. Additionally, there was a trend towards a significant correlation between Stop-relevant Go-trial accuracy and pro-active response slowing $(r=0.4 ; p=0.07)$. Finally, participants who had a higher proportion of successful Stop-trials displayed more pro-active response slowing $(r=0.84 ; p<0.001)$ and more progressive response slowing $(r=0.66 ; p=0.004)$, whereas none of the other correlations among this set of variables reached significance (Table 2). 
Table 2

behavior-behavior correlation coefficients.

\begin{tabular}{|c|c|c|c|c|c|c|}
\hline & SSRTm & SSRTi & $\begin{array}{l}\text { Correct } \\
\text { Stop-relevant } \\
\text { Go-trials }\end{array}$ & $\begin{array}{l}\text { Proportion } \\
\text { successful } \\
\text { stops }\end{array}$ & $\begin{array}{l}\text { Progressive } \\
\text { response } \\
\text { slowing }\end{array}$ & Pro-active response slowing \\
\hline SSRTm & - & & & & & \\
\hline SSRTi & $0.67^{* *}$ & - & & & & \\
\hline Correct Stop-relevant Go-trials & $-0.81^{* * *}$ & -0.33 & - & & & \\
\hline Proportion successful stops & $-0.49^{*}$ & 0.19 & $0.64^{* *}$ & - & & \\
\hline Progressive response slowing & $-0.38^{(*)}$ & 0.09 & $0.62^{* *}$ & $0.66^{* *}$ & - & \\
\hline Pro-active response slowing & -0.25 & 0.34 & $0.4^{(*)}$ & $0.84^{* * *}$ & $0.39^{(*)}$ & - \\
\hline
\end{tabular}

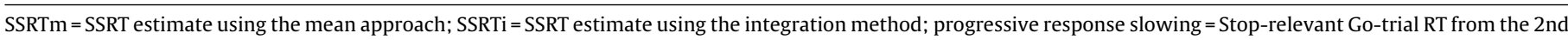
half of the experiment minus that of the first. Pro-active response slowing = Stop-relevant Go-trial RT minus Stop-irrelevant Go-trial RT.

${ }^{(*)} p<0.1$.

$p<0.05$.

** $p<0.01$.

*** $p<0.001$.

In light of this overall set of correlational results with the two SSRT measures, a consistent pattern appears to emerge. Specifically, the strategic slowing on Go-trial responses is related to a higher proportion of successful Stop-trials, higher Go-trial accuracy, and a faster SSRTm estimate. In contrast, the SSRTi estimate was not significantly correlated with either of these parameters, presumably indicating that it is indeed more robust against subtle influences of strategic response slowing.

\subsection{Brain-behavior correlation}

Fig. 3 provides an overlay of the grand-average results for the conjunction contrast of successful and unsuccessful Stop-trials vs. Stop-irrelevant Stop-trials. The activated areas isolated in this contrast included, among some others, the left and right IFG protruding into the insula, the pre-SMA, the caudate nucleus, and the thalamus (see Table 5 in [18] for the full list of 13 local activation maxima). As detailed in our previous report on this dataset, only a local maxima in the left anterior insula correlated with the SSRTm $(r=-0.69$; $p=0.002$ ), which also correlated with Stop-relevant Go-trial accuracy $(r=0.66 ; p=0.004)$.

The pattern of results of our present reanalysis of this brain-behavior data contrasts with the dramatic effects on behavior-behavior correlations. Specifically, the correlation between brain activity in the left anterior insula and stopping efficacy that we reported before was still significant when using the SSRTi $(r=-0.58 ; p=0.011)$, and the correlation coefficients did not differ significantly between the SSRTi and the SSRTm (two-tailed $p=0.55$ ). For the SSRTi, the correlation with the local maximum in the left thalamus also reached significance, but only if not corrected for multiple comparisons $(r=-0.52 ; p=0.047)$, whereas no other areas displayed correlations with the SSRTi (all $p>0.05$ ). Thus, among the set of activated areas in the investigated contrast, the SSRT estimation procedure appeared to have little influence on correlations between brain activity and SSRT. It is important to point out, however, that the present analysis was based on a relatively specific contrast that identified only a small set of areas, so that our conclusions concerning brain-behavior correlations with SSRTi and SSRTm are limited to these areas. ${ }^{2}$

\footnotetext{
2 Note also that the activity estimates used here are based on the average of successful and unsuccessful Stop-trials. Moreover, activity estimates for task-irrelevant Stop-trials were subtracted from both estimates. While the latter was done to enhance the specificity of the resulting values (i.e., in order to exclude activity unrelated to response inhibition), the use of both SST and UST might seem more unconventional. However, due to our use of a very small stepsize in the staircase titration procedure, it is likely that SST and UST trial types produced substantial and similar levels of stop-related activity (see [18] and Section 4 below for further
}

\section{Discussion}

The present study investigated the influence of two different procedures of estimating stopping efficacy (i.e., the SSRT) in the Stop-signal task on behavior-behavior and brain-behavior correlations. We found that two widely used estimation procedures strongly influenced behavior-behavior correlations despite the fact that their grand-average values across subjects were nearly identical. In our previous report derived from the present data set, we reported that the SSRT and task-accuracy measures were strongly inversely correlated [18]. Moreover, we had found that the SSRT and task-accuracy parameters correlated with activity in the left anterior insula. These observations appeared to suggest that the SSRT did not specifically index different degrees of inhibitory ability, as previously thought, but might rather relate to more general factors such as motivation [17,23]. Our previous analyses, however, were based on the SSRTm estimate, which generally requires an even ratio of successful and unsuccessful Stop-trials for each participant $[15,16]$, a criterion that was not precisely met for all individual subjects. As noted earlier, it has been demonstrated that deviations from this ratio, which can arise from the tendency of participants to strategically slow down responses over the course of the experiment, can substantially affect the estimate of the SSRT [17].

Although in the current study the ratio between successful and unsuccessful Stop-trials was very close to 50/50 averaged across subjects, it ranged between $46 \%$ and $59 \%$ for the successful Stop-trials, varying somewhat across participants. It is therefore conceivable that the use of the SSRTm estimate inflated the correlation with Go-trial accuracy that we have recently reported [18], in that response-slowing could have simultaneously led to shorter SSRTm estimates and to higher Go-trial accuracy. To probe this possibility here, we recomputed the SSRT using the integration approach (SSRTi), which is less susceptible to deviations from exactly even ratios of successful and unsuccessful Stop-trials. Although the average SSRT estimates across subjects did not differ significantly between the two procedures (SSRTm: $230 \mathrm{~ms}$; SSRTi: $225 \mathrm{~ms}$ ), the correlation pattern with other parameters differed quite substantially. In particular, the correlation with Go-trial task accuracy was indeed substantially attenuated when using the SSRTi and was not significant, in contrast to this correlation being highly significant when using the SSRTm. Moreover, the calculated coefficients for the correlations of accuracy and SSRT when using the SSRTi vs. the SSRTm were significantly different from each other. Thus, it seems likely that the correlation between SSRTm and

details). Moreover, when splitting up the brain-behavior correlations, we found that they were similar for the parameters based on SST and UST. 


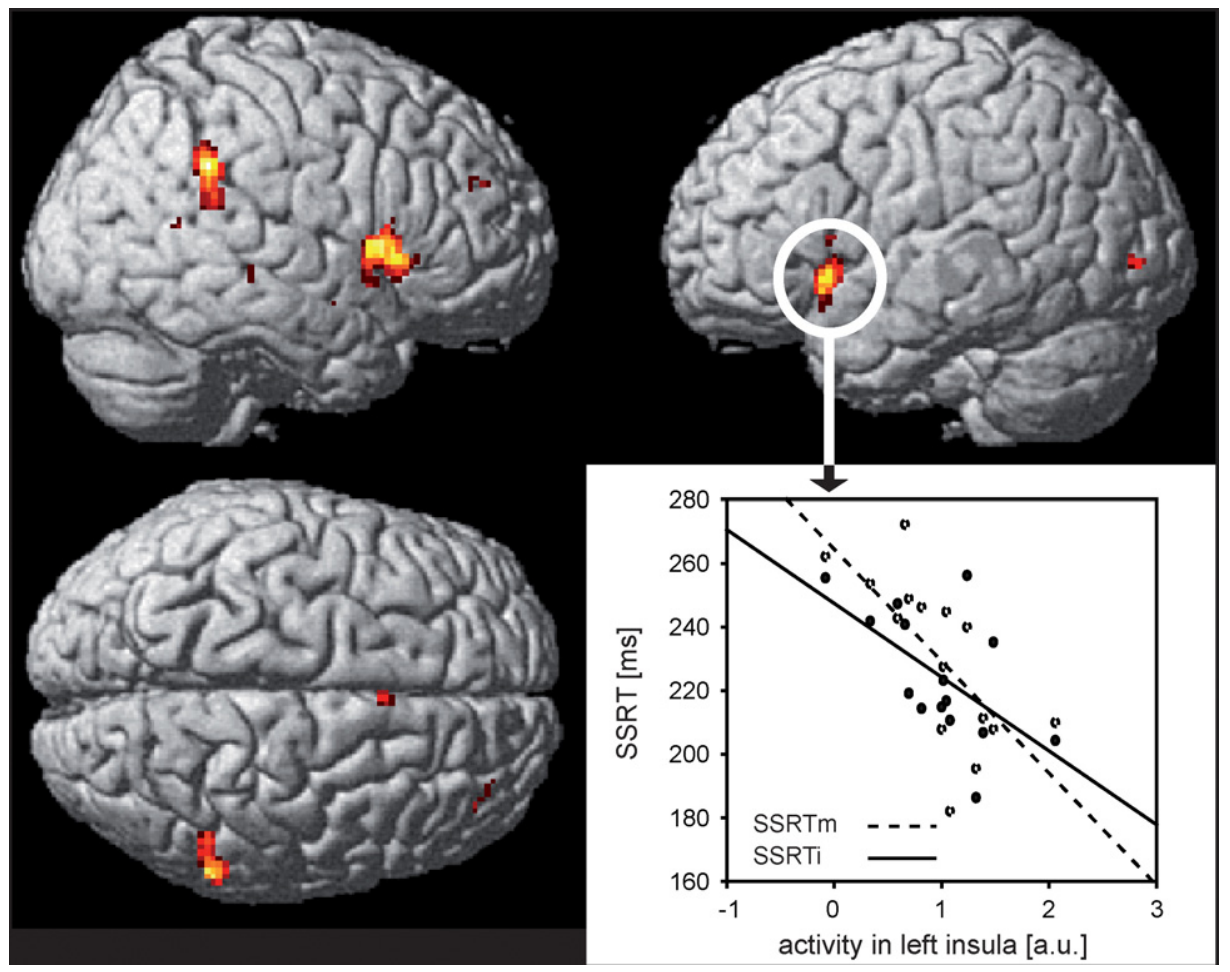

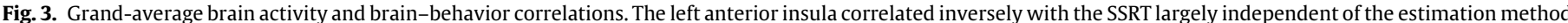

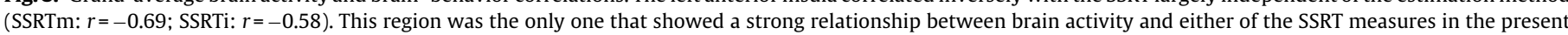
study (a.u. = arbitrary units of parameter estimates).

task accuracy was indeed inflated by different degrees of strategic response slowing across participants.

Further corroboration for this notion came from additional behavior-behavior correlations. Specifically, the correlations including the ratio of successful Stop-trials, Go-trial accuracy, and the two parameters describing different aspects of strategic response slowing (pro-active and progressive) indicated that these parameters are highly correlated with one another and with the SSRTm but not with the SSRTi. Accordingly, it appears quite likely that progressive response slowing in particular (i.e., slowing over the course of the experiment) leads to higher rates of successful Stop-trials, higher Go-trial accuracy, and shorter SSRTm estimates. Taken together, these observations would argue against our previous suggestion that the SSRT might reflect more general processes rather than being specific for response inhibition abilities (see also [17,24-27]). Our results rather appear to indicate that the SSRT, when calculated suboptimally, can be problematically influenced by other behavioral variables. In particular, it appears that individual-subject SSRTm measures can be influenced by strategic response slowing by the participants even if it only occurs to a very moderate degree. Thus, this estimation approach should indeed only be used if the known prerequisite of an even ratio of successful and unsuccessful Stop-trials is quite precisely met. This is particularly relevant when the estimates are used for correlational analyses, because it would appear, at least in our data, that it is more the distributions of individual values that are affected, without significantly changing the average value. The SSRTi, in contrast, appears to be relatively robust to the influence of strategic response slowing, at least when the latter only occurs to a moderate degree, in that it did not display a significant correlation with any of the RT-slowing-related parameters and task-accuracy analyzed here.
Given that the SSRTi can also be calculated quite easily, and that it generalizes to various versions of the Stop-signal task and a wider range of stopping-success rates (i.e., beyond 50\%), the SSRTi would seem to have considerable potential as a technique that can be applied in a great number of experimental contexts. However, it should to be noted that other approaches exist, which may have some advantages in certain circumstances [16].

It is important to note that the influence of the SSRT estimation procedure on behavior-behavior correlations was stronger than one might have expected. In particular, the mean values across subjects from the two procedures were virtually identical, and the mean ratio between successful and unsuccessful Stoptrials was fairly close to $50 \%$ (52.7\%), in which case the use of the mean approach would appear to have been warranted. Along similar lines, in the study by Leotti and Wager that investigated the influence of response slowing on SSRT estimates, the group that was defined as "non-compliant" (i.e., displaying too much response slowing) had a ratio of successful Stop-trials of $69 \%$ in their first experiment, and also the amount of RT slowing was substantial in this experiment (more than $100 \mathrm{~ms}$ over the course of the experiment and more than $200 \mathrm{~ms}$ compared to a block with speeded Go-trials only) [17]. The present set of participants was far more homogenous and displayed much less evidence of strategic response slowing, so that only subtle influences on the SSRTm estimate would have been expected. Thus, the strong influence of the estimation approach on some of the behavior-behavior correlations was somewhat surprising.

The only brain-behavior correlation with SSRTm we had found in our earlier report was with the left anterior insula, activity in which also correlated with general task accuracy [18]. This correlation was replicated in the present analysis for the SSRTi, 
whereas no strong correlations were found in other areas for either estimate. Accordingly, in contrast to the substantial influence on behavior-behavior correlations, the left anterior insula activation does indeed appear to be related to the SSRT, independent of the SSRT estimation procedure. The additional correlation of activity in this area with task accuracy that we reported previously, however, still suggests a relatively unspecific role for this area in enabling good task performance that is not specific for response inhibition (see also [11]).

Interestingly, the left anterior insula is not commonly identified in brain-behavior correlations with the SSRT. More generally, it can be noted that in the various studies that have investigated such brain-behavior correlations, the areas in which brain activity has been reported to correlate significantly with the SSRT have varied greatly, with limited overlap across the studies [28-37]. This variation might be related to power issues and the high dimensionality of the employed brain measures [38]. Additionally, other design factors or the use of different functional contrasts for quantifying activity related to response inhibition might have played a role in producing such variation. For example, the present study used activity estimates from a conjunction of successful and unsuccessful Stop-trials (relative to the Stop-irrelevant control condition), as the two conditions yielded very similar activation patterns. This latter finding could be related to a race model where inhibition gets triggered for successful and unsuccessful Stop-trials, so that activity related to response inhibition should be present in both conditions [18]. Additionally, however, the SSRT estimation approach employed might have played a role in the correlation variations across studies, in that we have shown in the present study that the estimation approach can strongly influence the across-subject distribution of SSRT values and thus the correlational analyses of these values.

Lastly, we note that although the reported brain-behavior correlation with activity in the left anterior insula was largely unaffected here, we only tested a small number of areas-i.e., only those that resulted from the highly specific contrast that we employed. Thus, we caution that our present findings indicating an estimationapproach independence of the correlation with this particular area therefore do not necessarily generalize to a wider set of brain areas. In particular, it would be conceivable that brain areas that are involved in strategic response slowing could also correlate with the SSRTm in a fashion that would in fact be confounded by the strategic response slowing itself, which could affect not only correlational analysis, but also between-group comparisons of the SSRT if the groups differ in this regard. In this context, it is important to note, however, that there have been some suggestions that strategic response slowing could in fact shorten the "real" SSRT $[39,40]$. Although such an influence would represent a violation of the independence assumption of the horse-race model [8], such effects are fully conceivable. The full set of behavior-behavior correlations, and the fact that the SSRTi did not correlate with either of our response-slowing parameters, however, appears to indicate that significant influences of strategic slowing on the "real" SSRT were likely not present here.

\section{Conclusions}

The present report investigated how two different SSRT estimation procedures, both of which are fairly widely used, can influence behavior-behavior and brain-behavior correlations. A surprisingly strong effect on behavior-behavior correlations between the SSRTm and Go-trial accuracy appeared to be mediated by strategic response slowing by the participants, which was not seen for the SSRTi estimate. On the level of brain-behavior correlations, only the left anterior insula correlated clearly with SSRT (and, in turn, with Go-trial accuracy), and in this case the specific estimation procedure had little influence on the correlation. Taken together, these results demonstrate that correlations between the SSRT and other parameters are susceptible to strategic response slowing if the SSRT is calculated with the oft-used mean approach. This drastic influence on correlations with other parameters stands in contrast to the fact that the overall grand-average SSRT estimates across subjects did not differ significantly between the two approaches. Accordingly, the present results suggest that the distribution of individual-subject SSRT values can have a strong effect on correlational measures that might not be expected based on the grand-average estimates. Thus, it would seem that these differences are important to take into consideration in Stop-signal tasks employing correlational analyses involving the SSRT measures. In general, the SSRTi estimate appears to be less prone to such influences and thus would seem to be preferred over the standard SSRTm, even when the pre-requisite of having exactly $50 \%$ stopping success for every participant is only slightly missed.

\section{Acknowledgments}

This research was supported by NIH grants R01-MH060415 and R01-NS051048 to M.G.W. and funds of the Deutsche Forschungsgemeinschaft (BO 3345/1-1) to C.N.B. The authors would like to thank Dr. Michael Scholz for technical support.

\section{References}

[1] Aron AR. The neural basis of inhibition in cognitive control. Neuroscientist 2007;13:214-28

[2] Williams BR, Ponesse JS, Schachar RJ, Logan GD, Tannock R. Development of inhibitory control across the life span. Dev Psychol 1999;35:205-13.

[3] Aron AR. Introducing a special issue on stopping action and cognition. Neurosci Biobehav Rev 2009;33:611-2.

[4] Aron AR, Durston S, Eagle DM, Logan GD, Stinear CM, Stuphorn V. Converging evidence for a fronto-basal-ganglia network for inhibitory control of action and cognition. J Neurosci 2007;27:11860-4.

[5] Verbruggen F, Logan GD. Response inhibition in the stop-signal paradigm. Trends Cogn Sci 2008;12:418-24.

[6] Groman SM, James AS, Jentsch JD. Poor response inhibition: at the nexus between substance abuse and attention deficit/hyperactivity disorder. Neurosci Biobehav Rev 2009;33:690-8.

[7] Logan GD, Cowan WB, Davis KA. On the ability to inhibit simple and choice reaction time responses: a model and a method. J Exp Psychol Hum Percept Perform 1984;10:276-91.

[8] Logan GD. On the ability to inhibit thought and action: a user's guide to the stop signal paradigm. In: Dagenbach D, Carr TH, editors. Inhibitory processes in attention, memory, and language. San Diego: Academic Press; 1994. p. 189-239.

[9] Swick D, Ashley V, Turken AU. Left inferior frontal gyrus is critical for response inhibition. BMC Neurosci 2008;9:102.

[10] Chambers CD, Garavan H, Bellgrove MA. Insights into the neural basis of response inhibition from cognitive and clinical neuroscience. Neurosci Biobehav Rev 2009;33:631-46.

[11] Swick D, Ashley V, Turken U. Are the neural correlates of stopping and not going identical? Quantitative meta-analysis of two response inhibition tasks. Neuroimage 2011;56:1655-65.

[12] de Jong R, Coles MGH, Logan GD, Gratton G. Searching for the point of no return: the control of response processes in speeded choice reaction performance. J Exp Psychol Hum Percept Perform 1990;16:164-84.

[13] Boucher L, Palmeri TJ, Logan GD, Schall JD. Inhibitory control in mind and brain: an interactive race model of countermanding saccades. Psychol Rev 2007; 114:376-97.

[14] Logan GD, Cowan WB. On the ability to inhibit thought and action: a theory of an act of control. Psychol Rev 1984;91:295-327.

[15] Band GPH, van der Molen MW, Logan GD. Horse-race model simulations of the stop-signal procedure. Acta Psychol 2003;112:105-42.

[16] Verbruggen F, Logan GD. Models of response inhibition in the stop-signal and stop-change paradigms. Neurosci Biobehav Rev 2009;33:647-61.

[17] Leotti LA, Wager TD. Motivational influences on response inhibition measures. J Exp Psychol Hum Percept Perform 2010;36:430-47.

[18] Boehler CN, Appelbaum LG, Krebs RM, Hopf JM, Woldorff MG. Pinning down response inhibition in the brain-conjunction analyses of the Stop-signal task. Neuroimage 2010;52:1621-32.

[19] Schmajuk M, Liotti M, Busse L, Woldorff MG. Electrophysiological activity underlying inhibitory control processes in normal adults. Neuropsychologia 2006;44:384-95. 
[20] Hinrichs H, Scholz M, Tempelmann C, Woldorff MG, Dale AM, Heinze HJ. Deconvolution of event-related fMRI responses in fast-rate experimental designs: tracking amplitude variations. J Cogn Neurosci 2000;12(Suppl. 2):76-89.

[21] Verbruggen F, Logan GD. Proactive adjustments of response strategies in the stop-signal paradigm. J Exp Psychol Hum Percept Perform 2009;35:835-54.

[22] Friston KJ, Fletcher P, Josephs O, Holmes A, Rugg MD, Turner R. Event-related fMRI: characterizing differential responses. Neuroimage 1998:7:30-40.

[23] Padmala S, Pessoa L. Interactions between cognition and motivation during response inhibition. Neuropsychologia 2010;48:558-65.

[24] Lijffijt M, Kenemans JL, Verbaten MN, van Engeland H. A meta-analytic review of stopping performance in attention-deficit/hyperactivity disorder: deficient inhibitory motor control. J Abnorm Psychol 2005;114:216-22.

[25] Alderson RM, Rapport MD, Kofler MJ. Attention-deficit/hyperactivity disorder and behavioral inhibition: a meta-analytic review of the stop-signal paradigm. J Abnorm Child Psychol 2007;35:745-58.

[26] Alderson RM, Rapport MD, Sarver DE, Kofler MJ. ADHD and behavioral inhibition: a re-examination of the stop-signal task. J Abnorm Child Psychol 2008;36:989-98.

[27] Kenemans JL, Bekker EM, Lijffijt M, Overtoom CC, Jonkman LM, Verbaten MN. Attention deficit and impulsivity: selecting, shifting, and stopping. Int J Psychophysiol 2005;58:59-70.

[28] Aron AR, Poldrack RA. Cortical and subcortical contributions to Stop signal response inhibition: role of the subthalamic nucleus. J Neurosci 2006;26:2424-33.

[29] Li CS, Huang C, Constable RT, Sinha R. Imaging response inhibition in a stop-signal task: neural correlates independent of signal monitoring and postresponse processing. J Neurosci 2006;26:186-92.

[30] Li CS, Yan P, Sinha R, Lee TW. Subcortical processes of motor response inhibition during a stop signal task. Neuroimage 2008;41:1352-63.
[31] Zheng D, Oka T, Bokura $H$, Yamaguchi S. The key locus of common response inhibition network for no-go and stop signals. J Cogn Neurosci 2008;20:1434-42.

[32] Rubia K, Smith AB, Taylor E, Brammer M. Linear age-correlated functional development of right inferior fronto-striato-cerebellar networks during response inhibition and anterior cingulate during error-related processes. Hum Brain Mapp 2007;28:1163-77.

[33] Aron AR, Behrens TE, Smith S, Frank MJ, Poldrack RA. Triangulating a cognitive control network using diffusion-weighted magnetic resonance imaging (MRI) and functional MRI. J Neurosci 2007;27:3743-52.

[34] Leung H-C, Common Cai W. Differential ventrolateral prefrontal activity during inhibition of hand and eye movements. J Neurosci 2007;27:9893-900.

[35] Chao HH, Luo X, Chang JL, Li CS. Activation of the pre-supplementary motor area but not inferior prefrontal cortex in association with short stop signal reaction time-an intra-subject analysis. BMC Neurosci 2009;10:75.

[36] Boecker M, Drueke B, Vorhold V, Knops A, Philippen B, Gauggel S. When response inhibition is followed by response reengagement: an event-related fMRI study. Hum Brain Mapp 2011;32:94-106.

[37] Sharp DJ, Bonnelle V, De Boissezon X, Beckmann CF, James SG, Patel MC, et al. Distinct frontal systems for response inhibition, attentional capture, and error processing. Proc Natl Acad Sci USA 2010;107:6106-11.

[38] Congdon E, Mumford JA, Cohen JR, Galvan A, Aron AR, Xue G, et al. Engagement of large-scale networks is related to individual differences in inhibitory control. Neuroimage 2010;53:653-63.

[39] Chikazoe J, Jimura K, Hirose S, Yamashita K, Miyashita Y, Konishi S. Preparation to inhibit a response complements response inhibition during performance of a stop-signal task. J Neurosci 2009;29:15870-7.

[40] Jahfari S, Stinear CM, Claffey M, Verbruggen F, Aron AR. Responding with restraint: what are the neurocognitive mechanisms. J Cogn Neurosci 2010;22:1479-92. 